\title{
Confirmation, refinement, and extension of a study in intrafraction motion interplay with sliding jaw motion
}

\author{
Michael W. Kissick, a) Sarah A. Boswell, Robert Jeraj, and T. Rockwell Mackie \\ Department of Medical Physics, University of Wisconsin Medical School, Madison, Wisconsin 53706-1532
}

(Received 1 February 2005; accepted for publication 21 April 2005; published 23 June 2005)

\begin{abstract}
The interplay between a constant scan speed and intrafraction oscillatory motion produces interesting fluence intensity modulations along the axis of motion that are sensitive to the motion function, as originally shown in a classic paper by Yu et al. [Phys. Med. Biol. 43, 91-104 (1998)]. The fluence intensity profiles are explored in this note for an intuitive understanding, then compared with Yu et al., and finally further explored for the effects of low scan speed and random components of both intrafraction and interfraction motion. At slow scan speeds typical of helical tomotherapy, these fluence intensity modulations are only a few percent. With the addition of only a small amount of cycle-to-cycle randomness in frequency and amplitude, the fluence intensity profiles change dramatically. It is further shown that after a typical 30-fraction treatment, the sensitivities displayed in the single fraction fluence intensity profiles greatly diminish. (C) 2005 American Association of Physicists in Medicine. [DOI: 10.1118/1.1935774]
\end{abstract}

\section{INTRODUCTION}

The classic paper by Yu et al. ${ }^{1}$ introduced a one-dimensional model to evaluate on-axis intensity variations due to the interplay between sliding jaw motion and respiratory motion. Target motion along the scanning axis was modeled as a sinusoid, and the relative fluence received by each point in the target was assumed proportional to the amount of time spent in the scanning field. This simplified problem setup is analogous to the interplay between respiratory motion and collimator leaf motion, ${ }^{2}$ or, in the case of helical tomotherapy, to the interplay between respiratory motion and superior/inferior couch travel across a slit beam. Of particular interest to the medical physics community are the observations of Yu et al. ${ }^{1}$ that the interplay may result in hot and cold spots in which the delivered fluence differs from the planned fluence by more than a factor of $4^{3,4}$ that the hot and cold spots are minimized when the jaw width is large and the scanning velocity is small relative to the respiratory amplitude and frequency, ${ }^{5,6}$ and that fractionation smooths the cumulative distribution. ${ }^{3}$

The goal of this study was to extend the procedure and calculations outlined in $\mathrm{Yu}$ et $a l^{1}{ }^{1}$ to parameters typical of helical tomotherapy: principally a slower scan speed. The investigation outlined in Sec. II led to the discovery that at least one figure in Yu et al. ${ }^{1}$ was a bit different than we calculated, as described in Sec. III. We also discovered that the fluence intensity modulations are sensitive to the introduction of small amounts of randomness at each cycle to the phase, amplitude, or frequency of the sinusoidal breathing motion. It was then recognized that these calculations could add to the ongoing discussion about tumor motion in general. In particular, we find that delivering multiple fractions with randomized phases of the oscillatory motion dramatically reduces the intensity modulations, in agreement with Yu et al. ${ }^{1}$ and Bortfeld et al. ${ }^{2}$

\section{METHODS}

We set out to understand the scanning/respiratory motion interplay by reproducing the results of Yu et al. ${ }^{1}$ with their same setup: a slit beam of $1 \mathrm{~cm}$ width exposing a sinusoidally oscillating target, significantly longer than the beam slit width such that modulations in the fluence along the tumor can be plotted. The respiratory motion was represented by a sinusoid of peak-to-peak amplitude $3 \mathrm{~cm}$ and frequency 0.25 $\mathrm{Hz}$. The modulations are described in Yu et al. ${ }^{1}$ as integrals of the fluence for only those times when a given voxel sees the beam, and the reader is referred to Yu et al. ${ }^{1}$ for details of this method. We wrote three independent programs based on this method, one in which the beam is scanning across the patient as illustrated by Fig. 2 of Yu et al. ${ }^{1}$ and the other two in the equivalent frame in which the beam slit is stationary, but the patient scans across the stationary beam. Individual voxels were followed as the fluence was delivered over time-simply adding a contribution, $\phi \cdot \Delta t$, where $\phi$ is the fluence rate and $\Delta t$ is the time step in which the voxel is in beam, or using a numerical bisection method to determine the times when the oscillating target crossed in and out of the beam. All three programs yielded consistent results.

\section{RESULTS}

Figure 1 is our reproduction of Fig. 3 of Yu et al., ${ }^{1}$ with spatial and temporal sampling at increments of $0.01 \mathrm{~cm}$ and $0.005 \mathrm{~s}$, respectively, which is approximately a factor of 3 finer than needed. Figure 1(a) illustrates the variations in intensity along the tumor when the scan speed is $0.5 \mathrm{~cm} / \mathrm{s}$, and matches Fig. 3(a) of Yu et al. ${ }^{1}$ Figure 1(b) illustrates the intensity variations in the case of a $1.0 \mathrm{~cm} / \mathrm{s}$ scan speed.

Figure 2 contains plots of position versus time for individual points within the target, providing an intuitive explanation for the shape of the fluence integral curves in Fig. 1. 

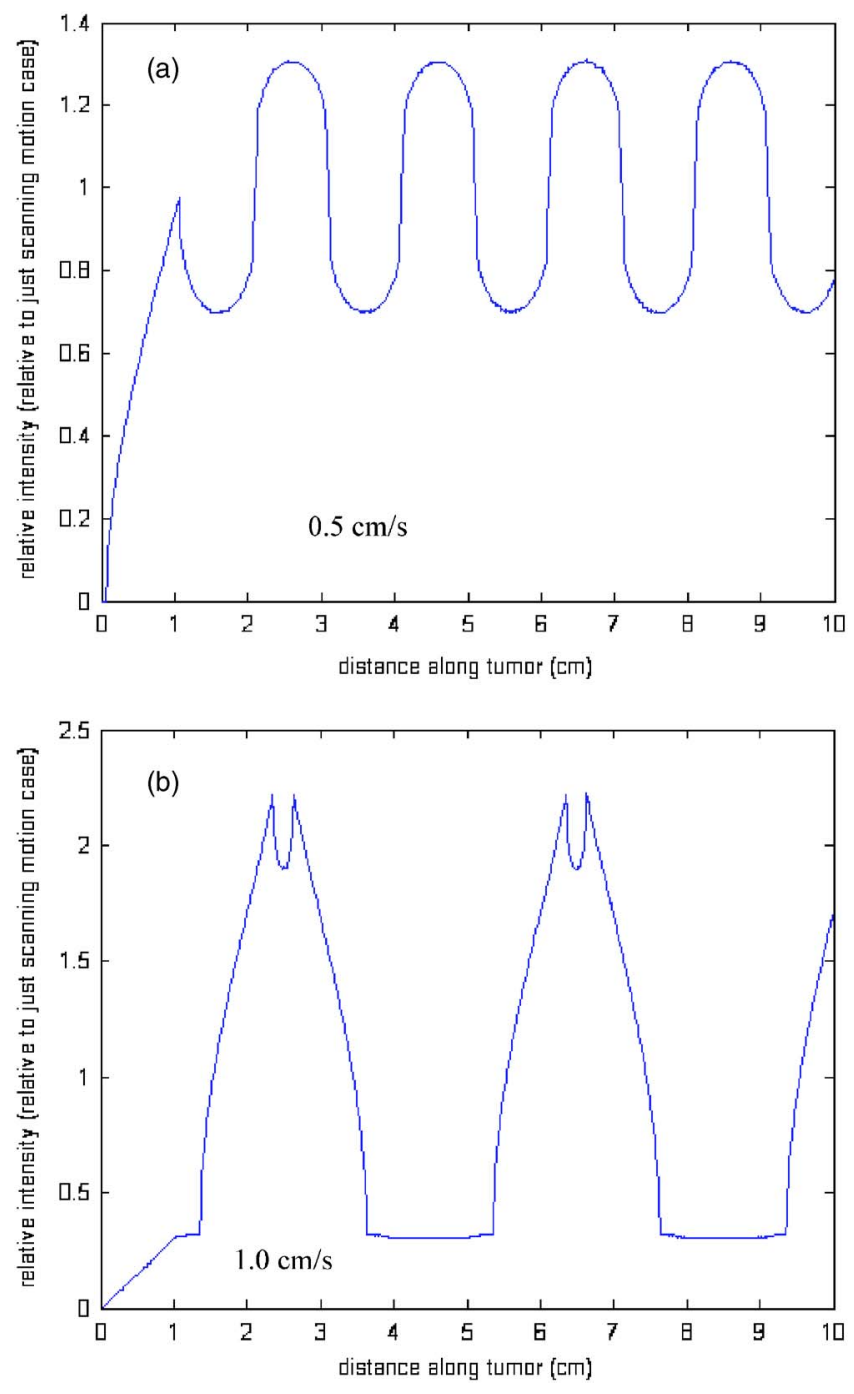

FIG. 1. Recreation of Fig. 3 of Yu et al. (Ref. 1) with our code. Intensity variation along the target due to interplay between respiratory motion of peak-to-peak amplitude $3.0 \mathrm{~cm}$ and frequency $0.25 \mathrm{~Hz}$, and scanning motion of a $1.0 \mathrm{~cm}$ beam slit at (a) $0.5 \mathrm{~cm} / \mathrm{s}$ and (b) $1.0 \mathrm{~cm} / \mathrm{s}$. As in all plots in $Y u$ et al., intensity variation is normalized to the intensity that would be received in the absence of respiratory motion. Note that (a) agrees with $\mathrm{Yu}$ et al. in magnitude and qualitatively, but (b) does not agree.

Points with distinct positions within the rigid-body target undergo the same one-dimensional breathing oscillation, superimposed on a constant scanning motion in the frame of the beam slit. In Figs. 2(a)-2(c), the $1 \mathrm{~cm}$ beam slit is shown superimposed on the motion curves of two or three illustrative points for scan speeds of $0.5,1.0$, and $2.0 \mathrm{~cm} / \mathrm{s}$, respectively. The total time that a tumor point spends between the horizontal lines that represent the beam slit is integrated to determine the relative intensity, which is plotted in Figs. 2(d)-2(f). Figures 2(d) and 2(e) are identical to Figs. 1(a) and 1(b), shown again for ease of comparison. The shape of the intensity profile was discovered to be shift invariant with respect to the phase of the sinusoidal oscillation.

Figure 3, an extension of Fig. 2, illustrates the motion of an individual point within the beam and the integrated intensity patterns for low scan speeds characteristic of helical tomotherapy. ${ }^{7}$ Here the scan speed is equivalent to the couch velocity. Moreover, we have confirmed the condition in $\mathrm{Yu}$ et al. ${ }^{1}$ for no intensity variation due to the interplay between sinusoidal respiratory motion and constant scanning motion in the case of $w=n(v / f)$ where $w$ is the slit width, $v$ is the scan speed, $f$ is the frequency of the oscillatory motion, and $n$ is an integer. For example, the fluence intensity at $0.25 \mathrm{~cm} / \mathrm{s}$ is constant across the target for the parameters used in Fig. 3.

An even more significant extension is to include realistic effects such as a random component to the oscillatory motion. The upper row of plots in Fig. 4 shows the motion of a point in the frame of the beam slit. The middle row shows the integrated fluence for a single fraction, and the bottom row shows the cumulative fluence over 30 randomly phased fractions. In the left column of Fig. 4, the target motion is a regular sinusoid superimposed on a constant scanning motion, so Fig. 4(g) is similar to Fig. 7(a) of Yu et al. ${ }^{1}$ In the middle and right columns, a random variation of up to $50 \%$ has been added to the amplitude and frequency, respectively, of each cycle of the target oscillation.

\section{DISCUSSION}

This note confirms many of the calculations in the classic treatment, while offering a refinement to at least one of these calculations. By independently coding the algorithm of $\mathrm{Yu}$ et al., ${ }^{1}$ we discovered an apparent computational error in their Fig. 3(b), which illustrates the relative intensity pattern along the axis of tumor motion for a single treatment fraction. We present a proposed corrected figure, with further insights to explain the shape of single and multifraction intensity patterns. The classic setup is also extended here to include the effects of randomized breathing amplitude or frequency on the cumulative intensity distributions.

Although the same jaw width, scan speeds, and breathing frequency and amplitude were used to create the fluence intensity profiles of Fig. 3 of Yu et al. ${ }^{1}$ and our Fig. 1, Fig. 3(b) of $\mathrm{Yu}$ et al. ${ }^{1}$ exhibits an asymmetric intensity pattern, whereas the pattern in our Fig. 1(b) is symmetric. The maximum intensity in Fig. 3(b) of Yu et al. ${ }^{1}$ appears to be about a factor of 1.8 more than the fluence intensity that would be received by the target in the absence of breathing motion, whereas in our Fig. 1(b), the maximum intensity is 2.2 times the fluence intensity that would be received in the absence of breathing motion. However, Fig. 1(a) matches Fig. 3(a) of Yu et al. ${ }^{1}$ We would always expect, at an intuitive level, that the fluence intensity modulation maxima should be symmetrically shaped. The reason is that, for a pure sine function, the peaks and the valleys have the same shapes and curvature magnitudes. If each cycle is identical and symmetric, then the modulation patterns would have to be as well.

Interplay of scanning and oscillatory motion produces several replications of an intensity modulation pattern within the target. It is evident from Fig. 2(b) that at fast scan speeds, variation of the fluence integral with position along the target is insignificant in the few centimeter quasilinear portion of 

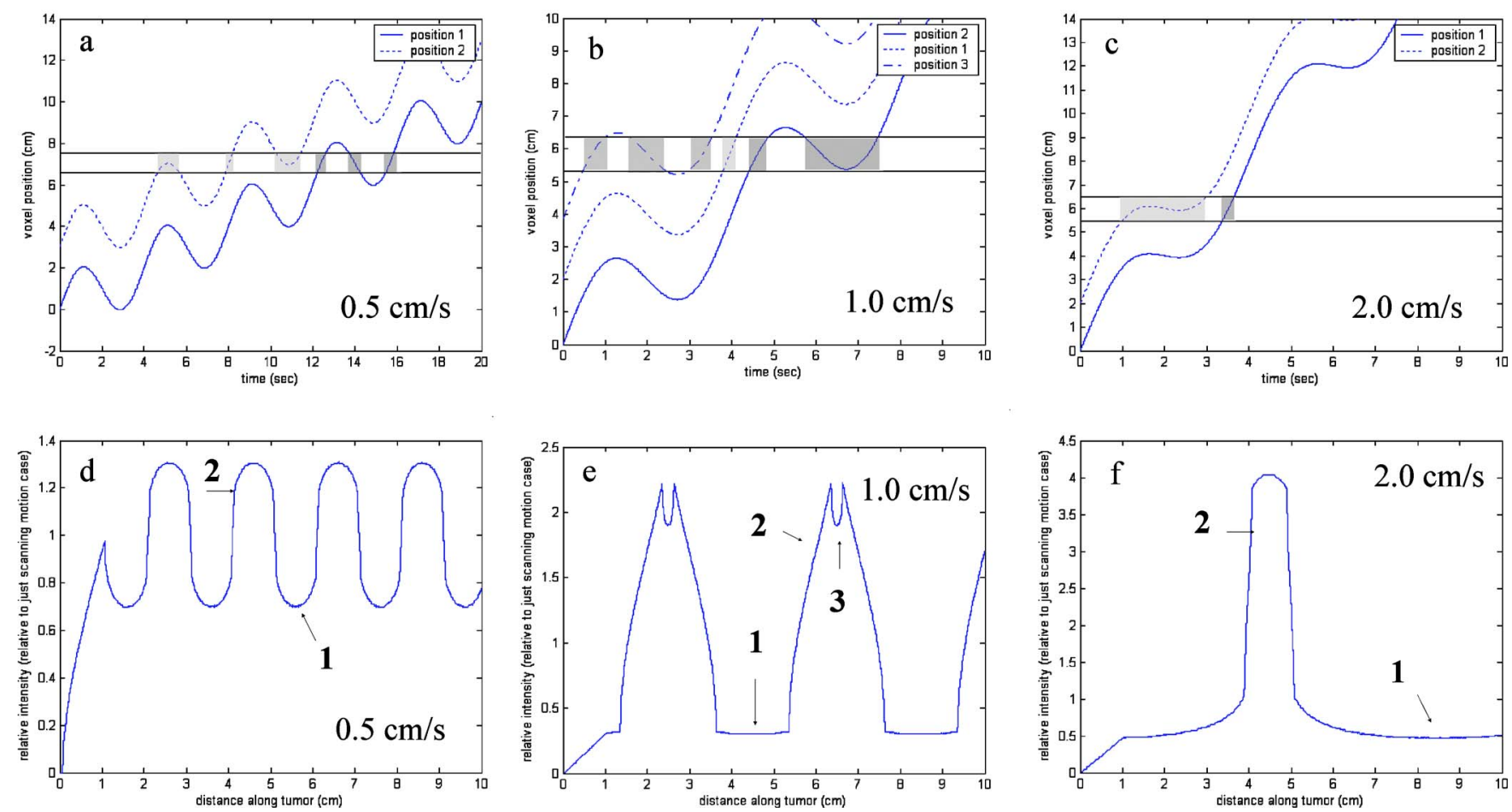

FIG. 2. Intuitive explanation of the intensity patterns in Fig. 1. Top row: the motion of two or three illustrative target points in the beam slit frame of reference. Points in the target have distinct starting positions, but follow the same motion pattern. A sinusoidal respiratory motion (frequency $0.25 \mathrm{~Hz}, 3 \mathrm{~cm}$ peak-to-peak amplitude) is superimposed on a constant scanning velocity of (a) $0.5 \mathrm{~cm} / \mathrm{s}$, (b) $1.0 \mathrm{~cm} / \mathrm{s}$, and (c) $2.0 \mathrm{~cm} / \mathrm{s}$. Horizontal lines indicate the $1 \mathrm{~cm}$ beam slits. Bottom row: integrated intensity values for each point in the target. Intensity values at the numbered positions in the bottom row of plots may be considered as integrals of the time spent in the beam by the correspondingly numbered illustrative curves in the top row. The plotted intensity is normalized to the intensity that would be received in the absence of respiratory motion.

the sinusoidal motion curve (position 1), but the variation suddenly becomes significant at the sinusoidal peaks and valleys (position 2). When the point is in just the right place to back out of the beam on both sides, then the fluence integral drops a bit (position 3). Therefore, sufficient temporal and spatial resolution is necessary to determine the correct shape of the fluence distribution as the peaks and valleys are sampled. Other speeds may result in very different sensitivities to resolution. For example, in the $0.5 \mathrm{~cm} / \mathrm{s}$ case [Fig. 1(a)], the valleys miss the beam just as the peaks enter the beam [see Fig. 2(a)]. It should be noted that changing the phase of the sinusoidal motion is equivalent to changing the beam slit position, so the intensity modulation pattern is shift invariant (apart from the ramping effect, described in the following); our code verifies the shift invariance when the time resolution is set high enough to obtain the correct fluence integral curves.

A ramping effect is observed at the ends of the tumor if the beam is turned on after the distal jaw passes over the proximal end of the oscillating tumor, or if the beam is turned off before the proximal jaw passes over the distal end of the oscillating tumor. This ramping is visible in the first few centimeters of the figures of Yu et al., ${ }^{1}$ since the proximal end of the tumor is aligned with the proximal jaw when the beam is turned on. Please note that in Figs. 2 and 3, the location of the beam slit is just for illustration. In the code, it was adjusted for each scan speed so that the distal end of the tumor was aligned with the distal jaw when the beam was turned off, thus allowing for the complete ramp up to start at position zero in the tumor. Our code used for the figures in this paper has the breathing patient scanning across the stationary beam as in tomotherapy, and it has the relative motion in the opposite direction of Yu et al. ${ }^{1}$ We have confirmed that this slight difference in approach does not affect the shape of the intensity pattern.

The intensity variation is less pronounced when slow scan speeds are used, since the target backs in and out of the beam many times. The other very interesting effect is that the convolution ramp-up extends along the tumor axis a distance equal to the beam width plus the peak-to-peak oscillation amplitude $[1 \mathrm{~cm}$ plus $3 \mathrm{~cm}$, see Fig. 3(d) where the ramp extends almost $4 \mathrm{~cm}$ along the tumor]. This larger convolution ramp-up implies a loss of resolution for delivery along the axis of motion. However, for the slow scan speeds typical of helical tomotherapy, the fluence intensity modulations become quite small even if no averaging or gating is considered. This is because the multiple backing in and out of the beam acts to average the fluence intensity variations together, thereby decreasing the integrated, resulting, fluence intensity modulations.

This paper restricts itself to one-dimensional longitudinal motion, the same dimension for typical lung motions. All of the intensity modulation in tomotherapy in the transverse plane with programmed leaf motions about many specific 

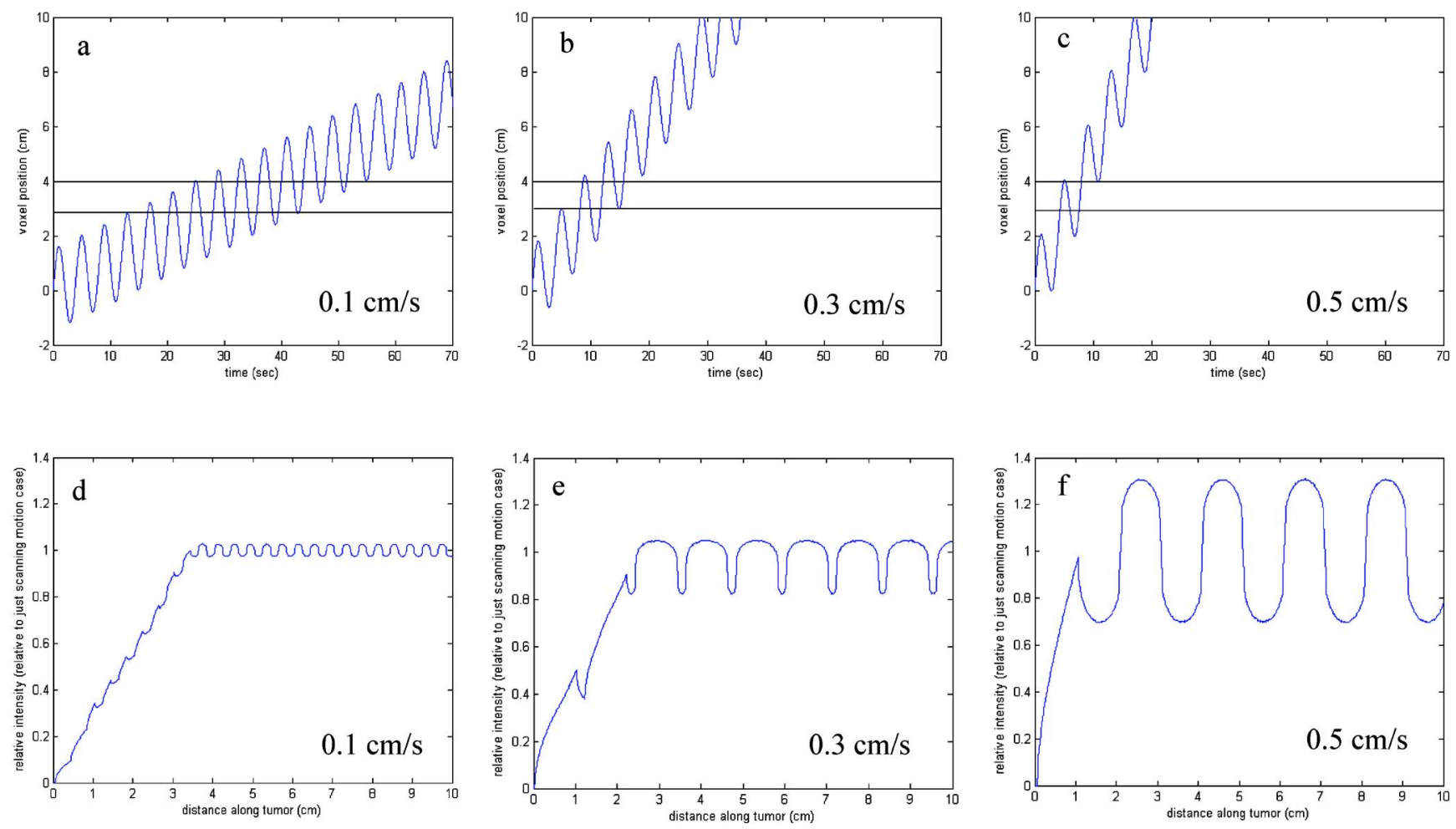

FIG. 3. Fluence intensity profiles for slow scan speeds typical of helical tomotherapy. Top row: motion of an illustrative point in the beam slit frame of reference. Sinusoidal motion of frequency $0.25 \mathrm{~Hz}$ and peak-to-peak $3 \mathrm{~cm}$ is superimposed on a constant scanning motion of velocity (a) $0.1 \mathrm{~cm} / \mathrm{s}$, (b) $0.3 \mathrm{~cm} / \mathrm{s}$, and (c) $0.5 \mathrm{~cm} / \mathrm{s}$. Horizontal lines indicate the $1 \mathrm{~cm}$ beam slits. Bottom row: corresponding intensity variation plots, normalized to the intensity that would be received in the absence of respiratory motion.

angles. In tomotherapy, the slice width is fixed in the longitudinal direction. The binary leaves in tomotherapy are switching from open to closed on the order of $50-100 \mathrm{~ms}$, which is much faster than the lung motion. The calculations in this paper would find applicability to helical tomotherapy for simple cases in discussing motion effects from longitudinal motion deep inside the tumor regardless of whether the planned dose in the axial dimension is uniform. The less variation that the local geometry has in the axial dimension, the more applicable these results would be to a complex situation involving helical tomotherapy.

We confirmed that the randomly phased addition of many fractions (Fig. 4) does reduce the variation as shown in Fig. 7 of Yu et al. ${ }^{1}$ In all three cases (constant frequency and amplitude, randomized amplitude, and randomized frequency), the averaging of many fractions similarly decreases the magnitude of the cumulative intensity modulations, but the cumulative intensity profile exhibits a repeating pattern in the case of constant frequency and amplitude [Fig. 4(g)]. The high sensitivity of single fraction fluence intensity profiles to small variations in the motion shown in (b) and (c) is evident. However, when many fractions are accumulated, it is hard to differentiate between the randomized amplitude and frequency cases. Other studies ${ }^{8}$ are also confirming the theories of Bortfeld et al.: ${ }^{2}$ realistic clinical cases display little motion influence within the clinical target volume (CTV) after a typical multifraction treatment.

\section{CONCLUSION}

Thus the general conclusions from $\mathrm{Yu}$ et al. ${ }^{1}$ are confirmed for the very idealized model. However, an interesting conclusion from our work in extending the groundbreaking efforts of Yu et al. ${ }^{1}$ is that some details in their relative intensity plots could have been sensitive to small computation errors. In particular, we believe that their Fig. 3(b) should look more like our Fig. 1(b). Slow scan speeds are one easy method to reduce the fluence modulations, with the unfortunate side effect of a larger ramp-up. Intra-fraction random components in the frequency or the amplitude have similarly dramatic effects on the fluence intensity profiles in each fraction. However, after a typical 30-fraction treatment, the magnitude of the dose intensity modulations diminishes similarly regardless of the presence or absence of random frequency or amplitude components from individual fractions.

\section{ACKNOWLEDGMENTS}

We are grateful for discussions with Dr. Cedric Yu. This work was made possible by a United States National Institute of Heath (NIH) Grant, United States National Cancer Institute Training Grant and NIH Grant (T32 CA09206 and PO1 CA88960). 

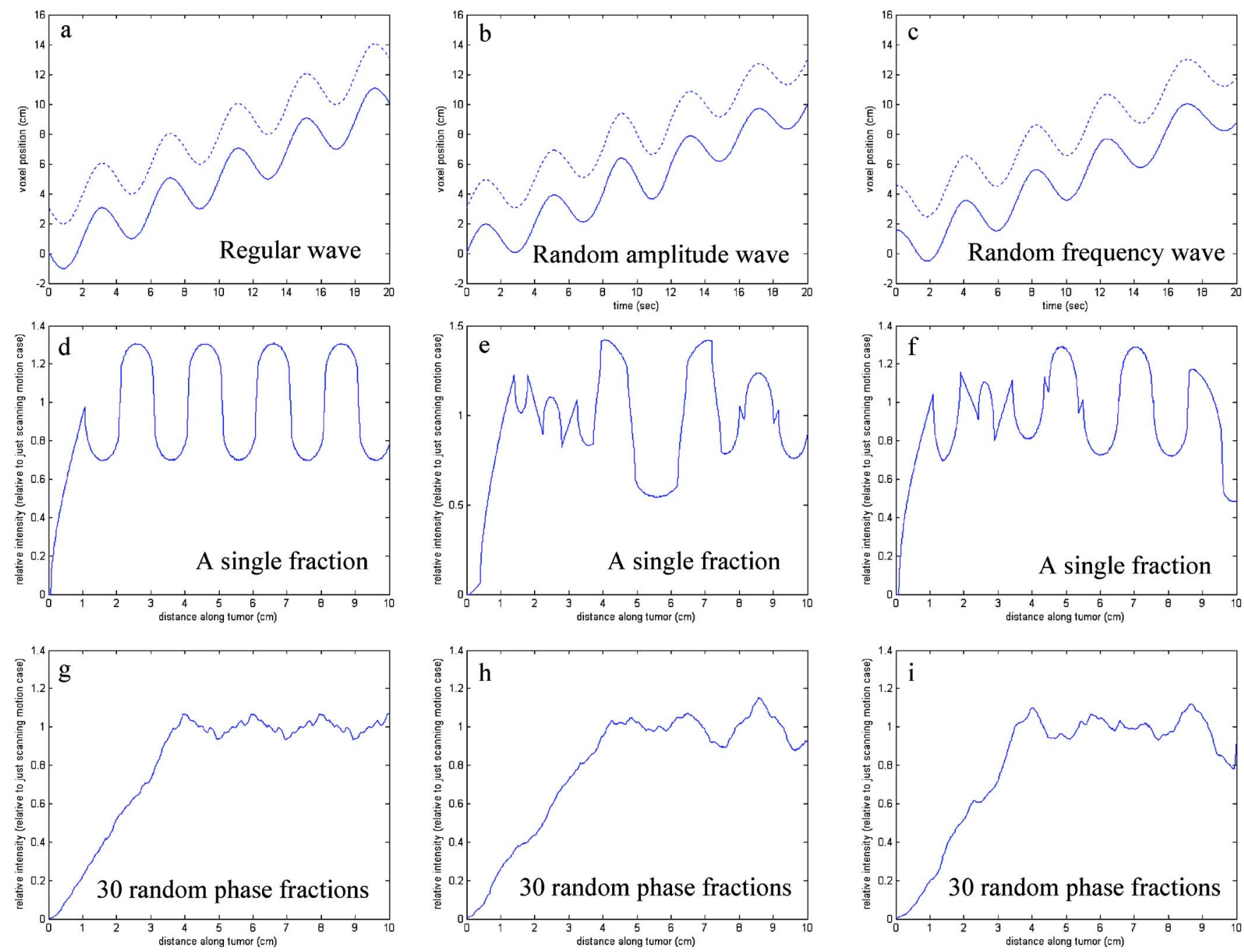

FIG. 4. Single and multifraction fluence intensity profiles with the introduction of random motion components. Top row: motion of illustrative points in the beam slit frame of reference: $0.25 \mathrm{~Hz}, 3 \mathrm{~cm}$ peak-to-peak sinusoidal motion added to $0.5 \mathrm{~cm} / \mathrm{s}$ constant motion in the case of (a) a regular wave, (b) up to $50 \%$ random amplitude variation at each cycle, and (c) up to 50\% random frequency variation at each cycle. Middle row: intensity variation plots for a single fraction of the motion depicted in the corresponding plots in the top row. Bottom row: plots (g)-(i) are cumulative intensity plots when summing 30 randomly phased fractions of the respective single-fraction curves in the middle row. The plotted intensity is normalized to the intensity that would be received in the absence of respiratory motion.

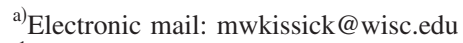

${ }^{1}$ C. X. Yu, D. A. Jaffray, and J. W. Wong, "The effects of intra-fraction organ motion on the delivery of dynamic intensity modulation," Phys. Med. Biol. 43, 91-104 (1998).

${ }^{2}$ T. Bortfeld, S. B. Jiang, and E. Rietzel, "Effects of motion on the total dose distribution," Semin Radiat. Oncol. 14, 41-51 (2004).

${ }^{3}$ P. J. Keall, V. R. Kini, S. S. Vedam, and R. Mohan, "Motion adaptive X-ray therapy: A feasibility study," Phys. Med. Biol. 46, 1-10 (2001).

${ }^{4}$ D. A. Low, M. Nystrom, E. Kalinin, P. Parikh, J. F. Dempsey, J. D. Bradley, S. Mutic, S. H. Wahab, T. Islam, G. Christensen, D. G. Politte, and B. R. Whiting, "A method for the reconstruction of four-dimensional synchronized CT scans acquired during free breathing," Med. Phys. 30, 1254-1263 (2003).

${ }^{5}$ M. Schaefer, M. W. Münter, C. Thilmann, F. Sterzing, P. Haering, S. E.
Combs, and J. Debus, "Influence of intra-fractional breathing movement in step-and-shoot IMRT," Phys. Med. Biol. 49, N175-N179 (2004).

${ }^{6}$ S. B. Jiang, C. Pope, K. M. Al Jarrah, J. H. Kung, T. Bortfeld, and G. T. Y. Chen, "An experimental investigation on intra-fractional organ motion effects in lung IMRT treatments," Phys. Med. Biol. 48, 1773-1784 (2003).

${ }^{7}$ T. R. Mackie, T. Holmes, S. Swerdloff, P. Reckwerdt, J. O. Deasy, J. Yang, B. Paliwal, and T. Kinsella, "Tomotherapy: A new concept for the delivery of dynamic conformal radiotherapy," Med. Phys. 20, 1709-1719 (1993).

${ }^{8}$ C.-S. Chui, E. Yorke, and L. Hong, "The effects of intra-fraction organ motion on the delivery of intensity-modulated field with a multileaf collimator," Med. Phys. 30, 1736-1746 (2003). 